\title{
Combating the burden of musculoskeletal conditions
}

\section{J S Smolen}

\section{Alliance against arthritis}

A re rheumatic diseases neglected by European politicians and healthcare payers? No other group of disorders affects more people than the conditions that rheumatologists diagnose and treat. No other group of disorders leads to higher prevalence of disability than the ailments rheumatologists look after. No other group of disorders leads to a higher financial burden than the diseases rheumatologists care for.

In a recently published report on the burden of musculoskeletal conditions, ${ }^{1}$ the director general of the World Health Organisation concluded, that "although the diseases that kill attract much of the public's attention, musculoskeletal or rheumatic diseases are the major cause of morbidity throughout the world, having a substantial influence on health and quality of life, and inflicting an enormous burden of cost on health systems". In that publication, the following data are presented: $(a)$ low back pain affects about $80 \%$ of people at some time of their life; (b) $40 \%$ of people over 70 years of age have osteoarthritis (OA) of the knee, with $25 \%$ of those with OA unable to perform daily activities of life and an additional 55\% having some disability; (c) $1.1 \%$ of Norwegians $>60$ years have rheumatoid arthritis; and $(d)$ about two million people world wide have hip fractures, usually associated with osteoporosis.

The most recent statistics on Austrian health care show that in 2001 musculoskeletal conditions led to 8.4 million days of sick leave (more than 1 day per person living in the country, from newborns to the aged); in comparison, cardiovascular conditions accounted for 1.7 million days of sick leave, and even respiratory disorders, which include flulike syndromes, did not top the rheumatic diseases. ${ }^{2}$

Thus, although musculoskeletal diseases strike more frequently, and are more severe and more costly than conditions of any other system, people with arthritis have to fight harder for access to the best care, even if it improves quality of life and prevents sick leave and disability, and scientists in the field of rheumatology have difficulties in finding an appropriate place in the European Community's research funding programmes. Thus the current Framework Program 6 of the EU's Research Directorate offers special actions for "combating" cardiovascular diseases, neurological disorders, diabetes, and cancer, but rheumatologists have to search for niches where they can place applications, competing against scientists from all other fields of medicine and basic research. And these niches apply mostly to highly specialised research - in particular, genomics. Needless to say, the funding for "combating major diseases" exceeds that for the other activities related to medicine, and musculoskeletal conditions are not regarded as "major diseases" by our European authorities. In the light of the major achievements of rheumatology research over the past decade both in basic and clinical research, achievements which have partly permeated into many other fields of medicine, this is hard to understand and unacceptable for the millions of people afflicted, as well as doctors caring for them.

\section{"Musculoskeletal conditions are not regarded as major diseases by the authorities"}

In the current issue of the Annals, Woolf et al, on behalf of the Arthritis Action Group, present their data on musculoskeletal pain in Europe focusing on perceptions of treatment. ${ }^{3}$ They disclose worrisome results: people with pain often do not seek medical help and those who receive medical help are often ill informed about treatment options or risks of treatments, despite the interest of physicians in improving their patients' quality of life. These observations call for actions to improve awareness and information among both the population, in general, and physicians. But they also confirm our realisation that despite major advances in some areas, we need more insights into the causes and pathogeneses of most of the rheumatic diseases, we need new diagnostic tools, including approaches to early recognition, and we need new and better remedies. In other words, we need more research and better transposition of research achievements into practice. The forthcoming annual EULAR Congress in Berlin will examine many of these issues.

As a consequence of the above dilemmas, EULAR calls for, and is initiating, a new action: Alliance Against Arthritis, AAA. This action will consist of Europewide activities, and as a starting event EULAR has organised a press conference and a patient meeting in Brussels in March, followed by similar national activities. It is the aim to achieve an increase in funding for rheumatology research, to improve European disability legislation, and to promote activities towards awareness of the rheumatic diseases and of today's therapeutic possibilities. Both nationally and at a European level, such actions will necessitate dedicated cooperation between the Scientific and Social Leagues, which are all represented in EULAR. Moreover, in these efforts EULAR will cooperate with the Arthritis Foundation of the United States, which likewise organises an Arthritis Summit in Washington, DC, in March 2004 with support from Members of Congress, including Senator Edward Kennedy. Their activity will lead to further US legislation, including increased funding expenses for rheumatology research.

Are rheumatic diseases neglected by European politicians in contrast with those in America? This seems to be the case, and the AAA needs to change such attitudes as widely as possible. If we don't struggle for our patients and our field-who will?

Ann Rheum Dis 2004;63:329

doi: 10.1136/ard.2004.022137

Correspondence to: Professor J S Smolen, Division of Rheumatology, Department of Internal Medicine III, Medical University Vienna and Lainz Hospital, Vienna, Austria A-1090; josef.smolen@wienkav.at

\section{REFERENCES}

1 WHO Scientific Group on the Burden of Musculoskeletal Conditions at the Start of the New Millennium. The burden of musculoskeletal conditions at the start of the new millennium: report of a WHO Scientific Group. Geneva: World Health Organisation, 2003.

2 Statistik Austria. Jahrbuch der Gesundheitsstatistik 2001. Vienna: Statistik Austria, 2003.

3 Woolf AD, Zeidler H, Haglund U, Carr AJ, Chaussade S, Cucinotta D, Veale DJ, et al. Musculoskeletal pain in Europe: its impact and a comparison of population and medical perceptions of treatment in eight European countries. Ann Rheum Dis 2004;63:342-7. 